\title{
ARTICLE
}

\section{Exogenous NADPH ameliorates myocardial ischemia-reperfusion injury in rats through activating AMPK/mTOR pathway}

\author{
Jiang Zhu ${ }^{1}$, Yi-fei Wang ${ }^{1}$, Xiao-ming Chai ${ }^{1}$, Ke Qian ${ }^{2}$, Ling-wei Zhang ${ }^{1}$, Peng Peng ${ }^{1}$, Pei-min Chen ${ }^{1}$, Jian-fang Cao ${ }^{1}$, Zheng-hong Qin ${ }^{2}$,
} Rui Sheng ${ }^{2}$ and Hong Xie ${ }^{1}$

\begin{abstract}
Our previous study shows that nicotinamide adenine dinucleotide phosphate (NADPH) plays an important role in protecting against cerebral ischemia injury. In this study we investigated whether NADPH exerted cardioprotection against myocardial ischemia/reperfusion (I/R) injury. To induce myocardial I/R injury, rats were subjected to ligation of the left anterior descending branch of coronary artery for $30 \mathrm{~min}$ followed by reperfusion for $2 \mathrm{~h}$. At the onset of reperfusion, NADPH $\left(4,8,16 \mathrm{mg} \cdot \mathrm{kg}^{-1} \cdot \mathrm{d}^{-1}, \mathrm{iv}\right)$ was administered to the rats. We found that NADPH concentrations in plasma and heart were significantly increased at $4 \mathrm{~h}$ after intravenous administration. Exogenous NADPH $(8-16 \mathrm{mg} / \mathrm{kg})$ significantly decreased myocardial infarct size and reduced serum levels of lactate dehydrogenase (LDH) and cardiac troponin I (cTn-I). Exogenous NADPH significantly decreased the apoptotic rate of cardiomyocytes, and reduced the cleavage of PARP and caspase-3. In addition, exogenous NADPH reduced mitochondrial vacuolation and increased mitochondrial membrane protein COXIV and TOM20, decreased BNIP3L and increased BCl-2 to protect mitochondrial function. We conducted in vitro experiments in neonatal rat cardiomyocytes (NRCM) subjected to oxygen-glucose deprivation/restoration (OGD/R). Pretreatment with NADPH (60,500 nM) significantly rescued the cell viability and inhibited OGD/Rinduced apoptosis. Pretreatment with NADPH significantly increased the phosphorylation of AMPK and downregulated the phosphorylation of mTOR in OGD/R-treated NRCM. Compound C, an AMPK inhibitor, abolished NADPH-induced AMPK phosphorylation and cardioprotection in OGD/R-treated NRCM. In conclusion, exogenous NADPH exerts cardioprotection against myocardial I/R injury through the activation of AMPK/mTOR pathway and inhibiting mitochondrial damage and cardiomyocyte apoptosis. NADPH may be a potential candidate for the prevention and treatment of myocardial ischemic diseases.
\end{abstract}

Keywords: NADPH; myocardial ischemia-reperfusion injury; apoptosis; AMPK; mTOR; Compound C

Acta Pharmacologica Sinica (2020) 41:535-545; https://doi.org/10.1038/s41401-019-0301-1

\section{INTRODUCTION}

Coronary atherosclerotic heart disease is one of the most common diseases that poses a serious threat to human health and is one of the leading causes of death in most countries [1]. The pathological basis of coronary heart disease is coronary artery stenosis or obstruction, leading to myocardial ischemia or necrosis. The main strategy for the clinical treatment of myocardial ischemia is to relieve angina symptoms and restore coronary blood flow. However, during patency of the coronary artery, the myocardium may be further damaged by a sudden increase in oxygen, a process that is known as $I / R$ injury [2]. Currently, the main interventions for myocardial I/R injury in coronary heart disease include surgery and drug treatment [3]. However, surgery has a limited time window, while existing drug treatment often has side effects, such as bleeding and arrhythmia. Therefore, there is an urgent need to develop more effective and safer drug treatments for myocardial I/R.
Reduced nicotinamide adenine dinucleotide phosphate (NADPH), also known as reduced coenzyme II, is a phosphorylated derivative of the 2'-position of the ribose ring attached to adenine in nicotinamide adenine dinucleotide $\left(\mathrm{NAD}^{+}\right)$[4]. $\mathrm{NADPH}$ is produced mostly through the pentose phosphate pathway in the body, and another $20-30 \%$ of NADPH is generated via the citric acid-pyruvate cycle [5]. NADPH is involved in a variety of anabolic processes, such as the synthesis of lipids and nucleotides $[6,7]$. NADPH acts as a hydrogen donor in many biochemical reactions and is involved in the biotransformation of drugs, poisons, and some sex hormones [8]. Another important biological function of NADPH is to provide redox capacity for the antioxidant system, thus protecting cells against oxidative stress [9]. In addition, NADPH is involved in ATP production. NADPH, together with NAD oxidized by the respiratory chain, maintains cellular energy homeostasis.

Previous studies in our laboratory have shown that exogenous $\mathrm{NADPH}$ supplementation showed short-term and long-term

\footnotetext{
'Department of Anesthesiology, the Second Affiliated Hospital of Soochow University, Suzhou 215006, China and ${ }^{2}$ Laboratory of Aging and Nervous Diseases, Department of Pharmacology, Jiangsu Key Laboratory of Translational Research and Therapy for Neuro-Psycho-Diseases, Jiangsu Key Laboratory of Preventive and Translational Medicine for Geriatric Diseases, College of Pharmacentical Science, Soochow University, Suzhou 215123, China

Correspondence: Rui Sheng (sheng_rui@163.com) or Hong Xie (hongx93044@126.com)

These authors contributed equally: Jiang Zhu, Yi-fei Wang
}

Received: 24 April 2019 Accepted: 16 August 2019

Published online: 27 November 2019 
neuroprotection in rodent and monkey stroke models [10-12]. NADPH not only reduced the infarct volume after stroke but also significantly decreased the long-term mortality and improved the neurological deficit. In addition, overexpression of G6PD in neurons in vivo and in vitro markedly reduced I/R injury. G6PD may exert neuroprotection through increasing the pentose phosphate pathway to generate NADPH [13]. Therefore, we hypothesized that exogenous NADPH may have protective effects against myocardial I/R injury. In the present study, we established in vivo and in vitro models to investigate the effects of NADPH on myocardial I/R injury and explore the possible mechanisms.

\section{MATERIALS AND METHODS}

Myocardial $\mathrm{I} / \mathrm{R}$ in rats

All animals were used according to guidelines for the use of animals, and the research protocol was approved by the Ethics Committee of Soochow University. Adult male rats $(250-300 \mathrm{~g})$ were purchased from the Experimental Animal Center of Soochow University. The myocardial ischemia model was created by ligating the left anterior descending branch of the coronary artery of rats as described previously [14]. The rats were anesthetized with an intraperitoneal injection of $5 \%$ pentobarbital sodium $(70 \mathrm{mg} / \mathrm{kg})$. After tracheotomy, a homemade endotracheal tube was inserted and attached to a small animal ventilator (breath rate: 50-60 rpm, tidal volume: $2 \mathrm{~mL} / 100 \mathrm{~g}$ ) (Taimeng Software Co., Ltd, Chengdu). The chest and pericardium were dissected, and the heart was exposed. Then, the left anterior descending branch of the coronary artery was ligated with a polypropylene 6-0 suture, which was passed through a small plastic tube. In the case of ischemia, the tube was tightened against the heart surface to occlude the left anterior descending branch. Intravenous catheters were used for drug administration. The myocardial ischemia-reperfusion model was induced by ischemia for $30 \mathrm{~min}$ followed by reperfusion for $2 \mathrm{~h}$. Blood pressures and electrocardiograms were monitored during I/R. A significant increase in the ST segment was detected by electrocardiogram to confirm the ischemia. The body temperature was maintained at $37^{\circ} \mathrm{C}$ using a thermostat (Taimeng Software Co., Ltd, Chengdu). Sham-operated rats underwent the same treatment without ligation of the left anterior descending branch of the coronary artery.

\section{Cell culture}

Neonatal rat cardiomyocytes (NRCMs) were acquired from Sprague-Dawley rats $1-3$ days after birth $[15,16]$ (Experimental Animal Center of Soochow University, certificate No 20020008, Grade II). Under sterile conditions, the hearts of neonatal rats were removed and placed in precooled phosphate buffered saline (PBS). The left ventricle was isolated, cut into pieces, and digested with $0.1 \%$ collagenase for $3 \mathrm{~min}$ each time, $3-4$ times in total. The cell suspension was then centrifuged, and the supernatant was discarded. High-glucose DMEM containing $10 \%$ FBS, $100 \mathrm{mg} / \mathrm{mL}$ streptomycin, and $100 \mathrm{U} / \mathrm{mL}$ penicillin was then added to the pellet to resuspend the cells, and placed in a $37^{\circ} \mathrm{C}$ incubator $\left(5 \% \mathrm{CO}_{2}\right.$, $95 \% \mathrm{O}_{2}$ ) for $45 \mathrm{~min}$. Afterward, the culture medium was transferred to another dish, and $0.1 \mathrm{mM}$ 5-bromo-2-deoxyuridine (BrdU) (Sigma) was added to inhibit noncardiomyocyte growth. The medium was changed after $48 \mathrm{~h}$. On day 3 , the cardiomyocytes were digested with $0.25 \%$ trypsin and counted. Then, the cell density was adjusted, and the cells were seeded in 96-well plates, 24-well plates, or $25-\mathrm{mm}$ dishes for subsequent experiments.

Oxygen-glucose deprivation and restoration (OGD/R) model Cells were washed twice with PBS and then replaced with HBSS $\left(\mathrm{CaCl}_{2} 1.7 \mathrm{mM}, \mathrm{KCl} 3.5 \mathrm{mM}\right.$, Hepes $10 \mathrm{mM}, \mathrm{NaHCO}_{3} 5 \mathrm{mM}, \mathrm{KH}_{2} \mathrm{PO}_{4}$ $0.4 \mathrm{mM}, \mathrm{NaCl} 116 \mathrm{mM}, \mathrm{MgSO}_{4} 0.8 \mathrm{mM}$, and $\mathrm{pH}$ 7.2-7.4). Then, the cells were placed into a hypoxic incubator chamber (BillupsRothenberg MC-101, USA) and treated with $95 \% \mathrm{~N}_{2}$ and $5 \% \mathrm{CO}_{2}$ at $37^{\circ} \mathrm{C}$ for $10 \mathrm{~h}$. After oxygen-glucose deprivation (OGD), HBSS was replaced with complete DMEM, and the cells were cultured under normal conditions.

NADPH pretreatment in vivo and in vitro

$\mathrm{NADPH}$ is unstable in an acidic solution, so saline was first adjusted to $\mathrm{pH} 8.0$ with $10 \% \mathrm{NaOH}$, and then NADPH was dissolved in the saline at $\mathrm{pH} 8.0$ to the desired concentration before administration. The NADPH solution was used as soon as possible. In the myocardial I/R injury model, NADPH (Sigma) at 4, 8 , and $16 \mathrm{mg} / \mathrm{kg}$ (N4, N8, and N16) and diltiazem (Sigma) at $5 \mathrm{mg} /$ $\mathrm{kg}$ were administered intravenously at the onset of reperfusion. The NRCMs were treated with HBSS containing different concentrations of NADPH during OGD. After OGD, the cells continued to be treated with medium containing the indicated concentration of NADPH. The rats or cardiomyocytes in the I/R or $\mathrm{OGD} / \mathrm{R}$ group were treated with the same volume of vehicle.

\section{Measurement of myocardial infarct size}

Infarct size was evaluated using Evans Blue and 2,3,5-triphenyltetrazolium chloride (TTC) double staining $2 \mathrm{~h}$ after reperfusion. The tube was tightened to block the blood flow for $30 \mathrm{~min}$. Then, $1 \mathrm{~mL}$ of patent blue was injected into the vein, and the heart was removed $2 \mathrm{~h}$ after reperfusion. The left ventricle was separated and placed at $-20^{\circ} \mathrm{C}$ for $20 \mathrm{~min}$. Then, the left ventricle was cut into six equal slices. Afterward, the heart slices were stained with $2 \%$ TTC for $15 \mathrm{~min}$ at $37^{\circ} \mathrm{C}$ and fixed in $4 \%$ formaldehyde overnight. The next day, the heart slices were divided into blue parts and red parts and weighed separately. The infarct volume was calculated using the following formula. Infarct size (IS) = the weight of the white part. Area at risk $(A A R)=$ the weight of the white part + the weight of the red part. IS/AAR = the weight of the white part/(the weight of the white part + the weight of the red part) $\times 100 \%$. AAR/LV $=$ (the weight of the white part + the weight of the red part)/left ventricular weight $\times 100 \%[17,18]$.

\section{Cell viability assay}

Cells were seeded in 96-well plates. After treatment, $10 \mu \mathrm{L}$ of cell counting kit (CCK-8; Dojindo, Kumamoto, Japan) reagent was added to a final volume of $100 \mu \mathrm{L}$ of culture medium per well, followed by incubation for another $2 \mathrm{~h}$. Absorbance was measured at $450 \mathrm{~nm}$ using a microplate reader (Gene Company Limited, Hong Kong, China) [19].

Measurement of cardiac troponin I (cTn-I)

Blood samples were collected from the internal jugular vein $4 \mathrm{~h}$ after myocardial I/R. The concentration of CTn-I in serum was determined according to the kit instructions (Elabscience Biotechnology Co., Ltd., Wuhan). The $O D$ value at $450 \mathrm{~nm}$ was measured within $15 \min [20,21]$

\section{Hoechst staining}

Nuclei were stained with Hoechst and observed under a fluorescence microscope (OLYMPUS IX71, Japan) according to the Hoechst staining kit (Beyotime Biotechnology Co., Ltd., Shanghai). Nuclear shrinkage and bright staining were considered apoptotic nuclei. The apoptosis rate was measured by the ratio of the number of apoptotic cells to the total number of cells $[4,5]$.

\section{Flow cytometry}

The apoptosis rate was measured by flow cytometry (BD ARAlll, USA) according to the instructions of the Annexin V-FITC/PI Apoptosis Detection Kit (Multi Sciences (Lianke Biotech Co., Ltd., Hangzhou). Briefly, cells were trypsinized, and centrifuged at 5000 $\mathrm{r} / \mathrm{min}$ for $5 \mathrm{~min}$. Then, the supernatant was discarded, and the pellets were resuspended in $1 \times$ diluted buffer. Annexin $\mathrm{V}(5 \mu \mathrm{L})$ and PI $(5 \mu \mathrm{L})$ were added to the cell suspension in the dark. The suspension was then detected by flow cytometry within $15 \mathrm{~min}$. 
The apoptosis rate was determined as the ratio of early apoptotic cells (PI-negative, FITC/annexin V-positive) and late apoptotic cells (FITC/annexin V-and PI-positive).

TUNEL analysis

Two hours after reperfusion, the myocardium of the ischemic area was dissected and fixed in $4 \%$ paraformaldehyde at $4{ }^{\circ} \mathrm{C}$ overnight, and then embedded in paraffin. TUNEL staining was performed as described (Beyotime Biotechnology Co., Ltd., Shanghai) [22]. Cardiomyocyte apoptosis in the myocardium was observed by confocal microscopy (Carl Zeiss, Germany).

\section{Measurement of NADPH concentration}

Normal rats were injected intravenously with NADPH $(16 \mathrm{mg} / \mathrm{kg})$. Blood samples $(500 \mu \mathrm{L})$ were collected in the period $5 \mathrm{~min}-4 \mathrm{~h}$ after NADPH administration. Then, the rats were euthanized, and the hearts were removed. NADPH concentrations in the plasma and myocardium were measured using an $\mathrm{NADP}^{+} / \mathrm{NADPH}$ assay kit (BioAssay Systems, Hayward, CA) following the manufacturer's directions. The $O D$ values of the samples and standards at $565 \mathrm{~nm}$ were measured at room temperature using a full-wavelength microplate reader.

\section{Transmission electron microscopy}

Two hours after reperfusion, the left ventricle was removed, cut into $1-\mathrm{mm}^{3}$ tissue blocks and fixed in $2.5 \%$ glutaraldehyde at $4{ }^{\circ} \mathrm{C}$. The fixed tissue samples were sent to Shanghai Jiaotong University for sectioning and staining. The ultrastructure of the myocardium was observed under a transmission electron microscope (JEOL, JEM-1230).

\section{Western blotting}

Cells and left ventricles were washed with precooled PBS. Then, the samples were lysed and homogenized in lysate $(10 \mathrm{mM}$ Tris$\mathrm{HCl}, \mathrm{pH} 7.4,150 \mathrm{mM} \mathrm{NaCl}, 1 \%$ Triton-100, 1\% sodium deoxycholate, $10 \%$ SDS, and $5 \mathrm{mM}$ EDTA), and total protein was extracted. A BCA Protein Assay Kit (Thermo Scientific, MA, USA) was used to quantify the protein concentrations. Proteins were separated on SDS-PAGE gels and transferred to nitrocellulose membranes. The membranes were blocked with $5 \%$ milk dissolved in TBST $(0.05 \%$ Tween-20). The membranes were then incubated with primary antibodies against GAPDH (1:1000; Biyuntian Biological Co., Ltd. Shanghai AF0006), PARP (1:1000; CST \#9542), Bcl-2 (1:500; Santa Cruz D2513), cleaved caspase-3 (1:1000; CST \#9661), caspase-3 (1:1000; Proteintech 66470-2-lg), TOM20 (1:1000; CST \#42406), COXIV (1:1000; CST \#11967), BNIP3L/Nix (1:1000; CST \#12396), mTOR (1:1000; CST \#2972), p-mTOR (1:1000; CST; \#5536), p-AMPK (1:1000; CST \#2535), and AMP-activated protein kinase (AMPK) (1:1000; CST \#2603) overnight at $4{ }^{\circ} \mathrm{C}$. Next, the membranes were incubated with secondary anti-mouse $\operatorname{lgG}$ or anti-rabbit IgG (1:10 000; Li-Cor Bioscience, Lincoln, NE, USA) for $2 \mathrm{~h}$ in the dark at room temperature. Images of the protein-antibody interactions were captured using an Odyssey infrared imaging system (Li-Cor Bioscience). GAPDH was used as the loading control.

Statistical analysis

The data were analyzed with GraphPad Prism 5.0 software. The results are shown as the mean $\pm \mathrm{SD}$. One-way ANOVA with Newman-Keuls post hoc test was carried out to compare data from multiple groups. The differences were considered significant when $P<0.05$.

\section{RESULTS}

NADPH reduced myocardial I/R-induced injury in rats

To investigate the role of NADPH in myocardial $I / R$, a rat model of myocardial $\mathrm{I} / \mathrm{R}$ was established by ligating the left anterior descending branch of the coronary artery. NADPH 4, 8, and
$16 \mathrm{mg} / \mathrm{kg}$ (N4, N8, and N16) and diltiazem $5 \mathrm{mg} / \mathrm{kg}$ (positive control) were injected at the onset of reperfusion. Serum lactate dehydrogenase (LDH) activity was measured at $2 \mathrm{~h}$ after reperfusion. The results showed that serum LDH levels were significantly higher in the I/R group than in the sham group $(P<0.001)$, while the $\mathrm{I} / \mathrm{R}+\mathrm{N} 16$ group had significantly lower serum LDH levels (compared with the I/R group, $P<0.001$ ) (Fig. 1b). Serum cTn-I concentrations were also examined at $4 \mathrm{~h}$ after reperfusion. The cTn-I levels were significantly higher in the I/R group than in the sham group $(P<0.01)$, and the $c T n-I$ levels were significantly lower in the $\mathrm{I} / \mathrm{R}+\mathrm{N} 16$ group than in the $\mathrm{I} / \mathrm{R}$ group $(P<0.05$, Fig. $1 \mathrm{C})$. The infarct size was assessed using Evans blue and $T C$ double staining at $2 \mathrm{~h}$ after reperfusion. There was no significant difference in the risk area (AAR) between the groups, but the infarct size was significantly higher in the $\mathrm{I} / \mathrm{R}$ group than in the sham group $(P<$ 0.001 ), while the infarct size was significantly lower in the $I / R+$ N16 group and the I/R + N8 group than in the I/R group $(P<0.01$, $P<0.05$ ). (Fig. 1a). These data indicate that NADPH can effectively alleviate myocardial I/R injury.

\section{NADPH protected cardiomyocytes from OGD/R injury}

NRCMs were treated with NADPH at the indicated concentrations and then subjected to the OGD/R model. The cell viability of cardiomyocytes was measured by CCK8 assay. The CCK8 data showed that cell viability was significantly lower in the OGD/R group than in the control group $(P<0.001)$. However, administration of NADPH at concentrations of $20 \mathrm{nM}-500 \mathrm{nM}$ significantly alleviated cell death, as evidenced by improved cardiomyocyte morphology (Fig. 2a) and increased cell viability (Fig. 2b). These data indicate that NADPH can also significantly protect against myocardial I/R injury in primary NRCMs.

Exogenously administered NADPH enters the heart

To determine whether exogenously administered NADPH can reach the heart, we examined the distribution of NADPH in the blood and heart. Normal rats were injected intravenously with NADPH (16 mg/kg). Blood samples were collected at 5, 10, 30, 120, and 240 min after NADPH administration. NADPH concentrations in the plasma and myocardium were measured using an $\mathrm{NADP}^{+}$/ NADPH assay kit. The results showed a significant increase in serum NADPH levels at 5 min after NADPH administration (from $8 \mu \mathrm{M}$ to $16 \mu \mathrm{M}, P<0.001$, Fig. 3a), and NADPH levels remained high at $4 \mathrm{~h}$ after administration $(17 \mu \mathrm{M}, P<0.001)$. The concentration of NADPH in the myocardium also increased significantly at $4 \mathrm{~h}$ after NADPH administration (from $2.5 \mu \mathrm{g} / \mathrm{mg}$ to $5 \mu \mathrm{g} / \mathrm{mg}, P<$ 0.05 , Fig. 3b). The above results suggest that NADPH may enter the myocardium to exert cardioprotective effects.

NADPH inhibited myocardial I/R-induced apoptosis in cardiomyocytes

We then asked whether NADPH has an effect on cardiomyocyte apoptosis induced by I/R injury. NRCMs were treated with NADPH and then subjected to OGD/R. Cells were harvested $24 \mathrm{~h}$ after reperfusion. The results of flow cytometry (Fig. 4a) showed that the apoptosis rate (early and late apoptosis) was significantly higher in the OGD/R group than in the control group $(P<0.001)$, and the apoptotic rate was significantly lower in the OGD/R $+\mathrm{N}(\mathrm{L})$ group than in the OGD/R group $(P<0.05)$. Cardiomyocyte nuclei were stained with Hoechst 33342 and observed by fluorescence microscopy. Similarly, the results showed (Fig. 4b) that compared with those in the control group, many cardiomyocytes in the OGD/R group showed apoptosis $(P<0.001)$, characterized by brightly stained nuclei, nuclear condensation, and fragmentation. NADPH treatment significantly reduced the number of apoptotic cells $(P<0.01)$. Moreover, myocardial apoptosis induced by $\mathrm{I} / \mathrm{R}$ in rats was determined using TUNEL staining. The green fluorescence in the myocardium was significantly enhanced in the I/R model, indicating an increase in apoptosis in cardiomyocytes, while 
a

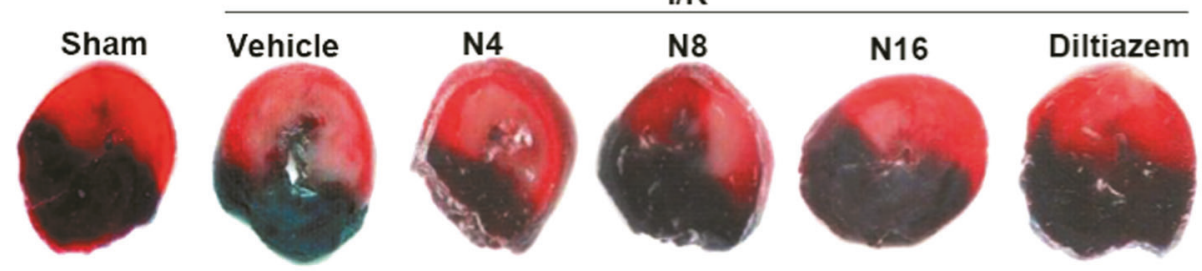

b

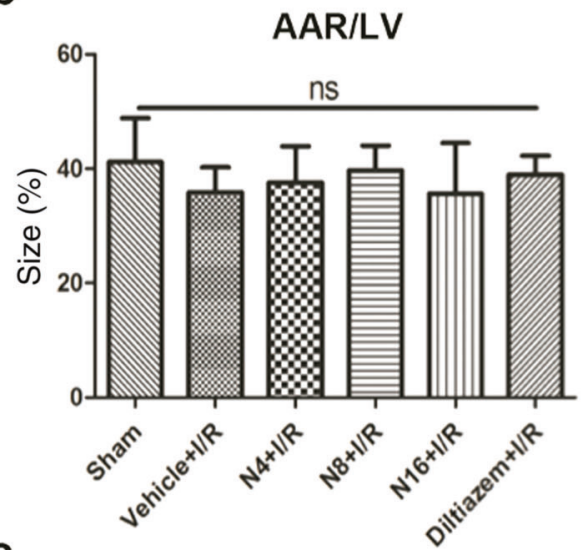

ISIAAR

C

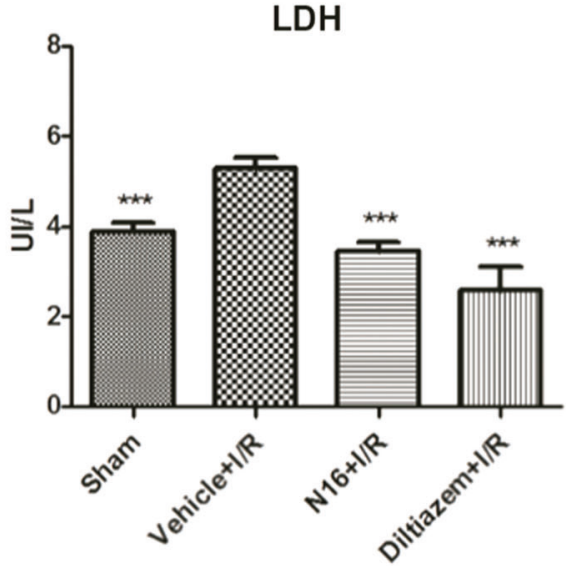

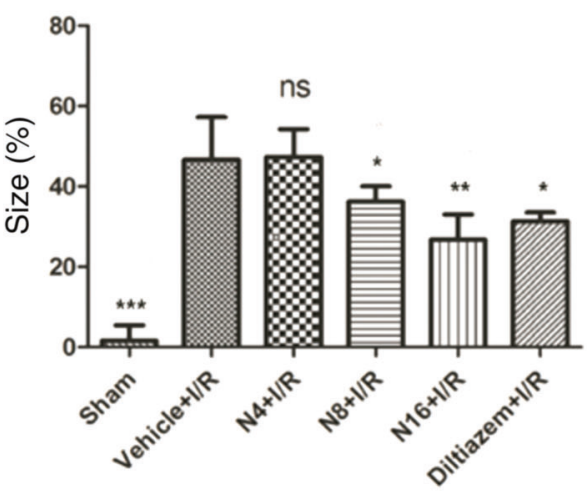

cTn-I

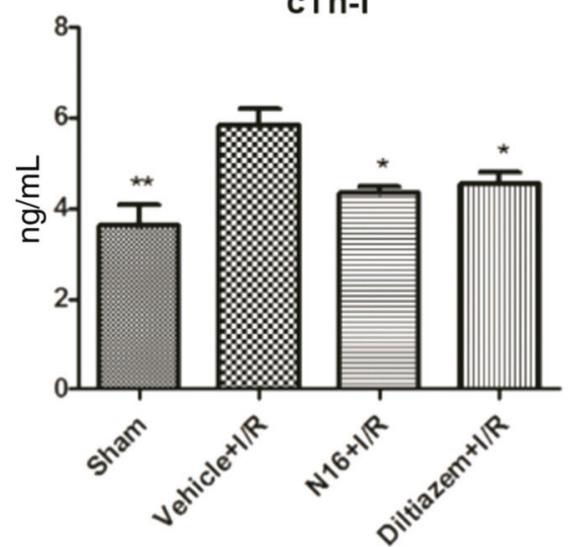

Fig. 1 NADPH reduced myocardial I/R-induced injury in rats. Myocardial I/R was established by ligating the left anterior descending artery in rats. NADPH 4, 8, and $16 \mathrm{mg} / \mathrm{kg}(\mathrm{N} 4, \mathrm{~N} 8$, and N16) and diltiazem were administered intravenously at the onset of reperfusion. The infarct size was evaluated $2 \mathrm{~h}$ after reperfusion. Representative photographs (a) and quantitative analysis (b) showed that NADPH decreased the infarct size. AAR area at risk, IS infarct size, LV left ventricle. c Cardiac function was evaluated by serum LDH at $2 \mathrm{~h}$ and $\mathrm{cTn}-\mathrm{I}$ at $4 \mathrm{~h}$ after reperfusion. LDH lactate dehydrogenase, cTn-I cardiac troponin I. $n=6 \sim 8$ rats. The bars represent the mean \pm SD, ${ }^{*} P<0.05,{ }^{* *} P<0.01$, ${ }^{* * *} P<0.001$ compared with the I/R group

NADPH significantly reduced the myocardial apoptosis (Fig. 5a). Western blotting was used to detect proteins involved in the apoptotic cascade. The results showed that OGD/R or I/R increased the cleavage of caspase- 3 and PARP in cardiomyocytes, while NADPH significantly reduced the cleavage of caspase- 3 and PARP (Figs. $4 c, d$, and 5b, $c, P<0.05$ or $P<0.01$ ). These results indicate that NADPH can significantly inhibit cardiomyocyte apoptosis induced by $\mathrm{I} / \mathrm{R}$ injury.

NADPH protects against I/R-induced mitochondria damage

Transmission electron microscopy was used to analyze the ultrastructure of the myocardium subjected to myocardial I/R. As shown in Fig. 6a, some of the myofilaments were lysed and disordered in the I/R group compared with those in the sham operation group. The cell membrane of some cardiomyocytes was destroyed. Mitochondria were severely damaged, and some mitochondria were vacuolated. The pathology of the myocardium in the NADPH group was significantly alleviated. The irregular arrangement of myofilaments and mitochondrial swelling and destruction were greatly improved, suggesting that NADPH may protect against myocardial injury and mitochondrial damage induced by I/R. BNIP3L regulates mitochondrial function by binding to $\mathrm{BCl}-2$ [23]. Western blot results (Fig. 6b) showed an increase in BNIP3L protein expression and a decrease in $\mathrm{Bcl}-2$ after I/R. After NADPH administration, the expression of BNIP3L decreased, while the expression of $\mathrm{Bcl}-2$ increased. Moreover, the expression of the mitochondrial membrane proteins COXIV and TOM20 indirectly represents the number and function of mitochondria $[24,25]$. The results showed that although $\mathrm{I} / \mathrm{R}$ had a tendency to reduce the expression of COXIV and TOM20, there was no significant difference between the sham-operated and I/R groups. However, NADPH treatment significantly increased the expression of COXIV and TOM20 (Fig. 6c, d). These data, combined with the mitochondrial ultrastructure and BNIP3L and $\mathrm{Bcl}-2$ results, suggested that I/R treatment impaired mitochondrial morphology and function, but it might not significantly affect the number of 
a

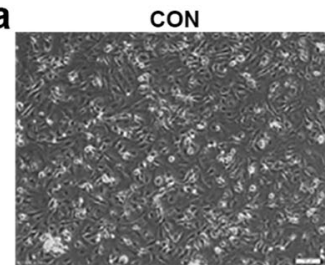

OGD/R+N(L)

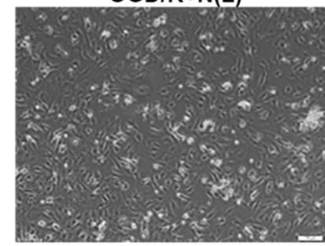

OGD/R

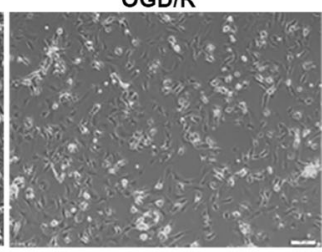

$\mathrm{OGD} / \mathrm{R}+\mathrm{N}(\mathrm{H})$

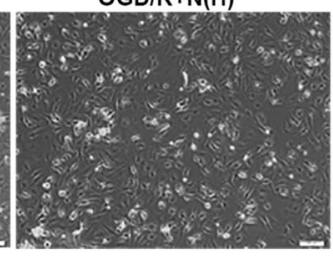

b

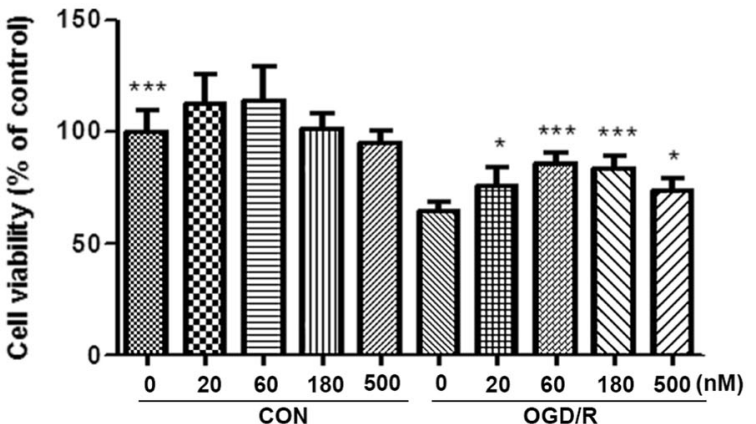

Fig. 2 NADPH protected cardiomyocytes from OGD/R injury. Neonatal rat cardiomyocytes were treated with NADPH at the indicated concentrations and then subjected to the OGD/R model. a Representative morphology of the cardiomyocytes. Bar $=100 \mu \mathrm{m}$. $\mathbf{b}$ Cell viability of the cardiomyocytes was measured by CCK8 assay. $n=3$ independent cultures. The bar represents the mean \pm SD, $P<0.05,{ }^{* * *} P<0.001$ compared with the OGD/R group

a

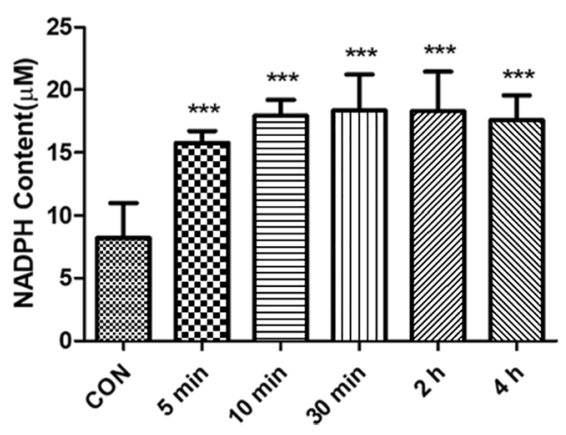

b

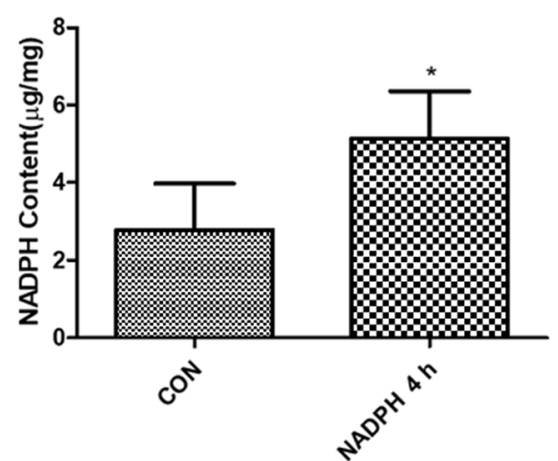

Fig. 3 Exogenous NADPH entered the heart. Normal rats were injected intravenously with NADPH (16 mg/kg). NADPH concentrations in plasma and myocardium were measured at the indicated time after NADPH administration. a NADPH concentration in the serum of rats. b NADPH concentration in the myocardium of rats. $n=6$ rats. The bar represents the mean $\pm \mathrm{SD},{ }^{*} P<0.05,{ }^{* *} P<0.01,{ }^{* * *} P<0.001$ compared with the CON group

mitochondria. Exogenous administration of NADPH can significantly improve the morphology and function of mitochondria and may also increase the number of mitochondria. The effect of $\mathrm{NADPH}$ on mitochondrial quality control remains to be further studied. These results suggest that NADPH may protect against mitochondrial damage induced by myocardial ischemia/ reperfusion.

NADPH activates the AMPK-mTOR pathway in myocardial I/R injury

To investigate whether NADPH shows cardioprotective effects through the AMPK-mTOR signaling pathway, we examined changes in protein expression by Western blotting. The results (Fig. 7a, b) showed that AMPK phosphorylation was reduced after OGD/R treatment compared with that in the untreated group $(P<$ $0.01)$. NADPH administration significantly increased AMPK phosphorylation and inhibited mTOR phosphorylation $(P<0.05)$. The results in the myocardium of rats (Fig. 7c, d) were consistent with those in NRCMs. Compared with phosphorylation in the sham group, AMPK phosphorylation was decreased $(P<0.01)$ and mTOR phosphorylation was increased in the I/R group. NADPH administration significantly reversed the above changes $(P<0.01)$.

Next, to further verify that NADPH functioned through the AMPK-mTOR signaling pathway, we used the AMPK inhibitor compound $C$ to inhibit AMPK activity. As shown in Fig. 8a, AMPK phosphorylation was partially abolished after treatment with compound $\mathrm{C}$ in both the $\mathrm{N}(\mathrm{L})+\mathrm{CpC}$ and $\mathrm{N}(\mathrm{H})+\mathrm{CpC}$ groups $(P<0.001$ vs. $\mathrm{N}(\mathrm{L})$ or $\mathrm{N}(\mathrm{H})$, respectively). Moreover, the cardioprotective effects of NADPH against $\mathrm{I} / \mathrm{R}$ were partially offset by compound $C(P<0.001$ vs. $N(L)$ or $N(H))$ (Fig. 8b), suggesting that NADPH may act by activating the AMPK pathway.

These data suggest that NADPH may inhibit cardiomyocyte apoptosis induced by I/R by activating the AMPK-mTOR signaling pathway.

\section{DISCUSSION}

Our previous studies have shown that NADPH has neuroprotective effects on cerebral ischemic injury through antioxidative and antiinflammatory effects [10-12] However, whether NADPH has an effect on myocardial ischemia has not been elucidated. Studies have shown that $\mathrm{NAD}^{+}$treatment significantly decreased myocardial infarct size, reduced serum troponin levels, and attenuated I/R-induced apoptosis in the myocardium [26]. In addition, Reyes et al. found that NADPH was markedly depleted in isolated rat hearts subjected to myocardial I/R [27]. Therefore, we assume that exogenous NADPH may have protective effects against myocardial I/R injury. In the present study, myocardial I/R injury was produced by ligating the left anterior descending branch of the coronary artery in rats. We found that exogenous 

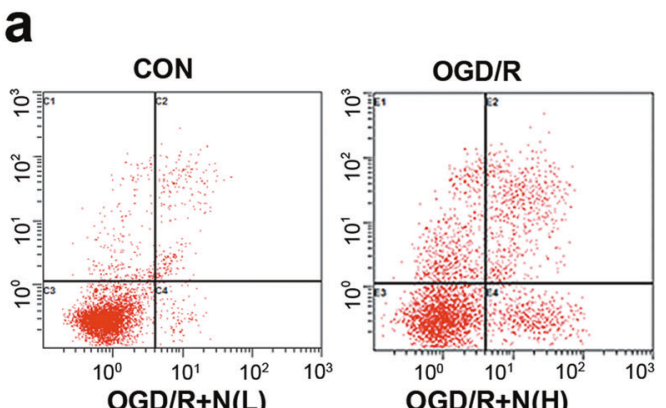

b $\cos$

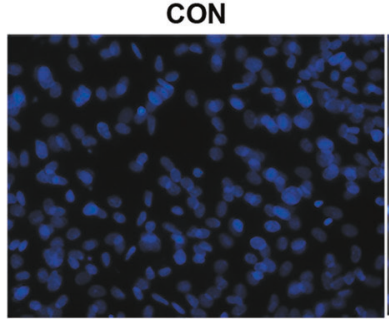

$O G D / R+N(L)$
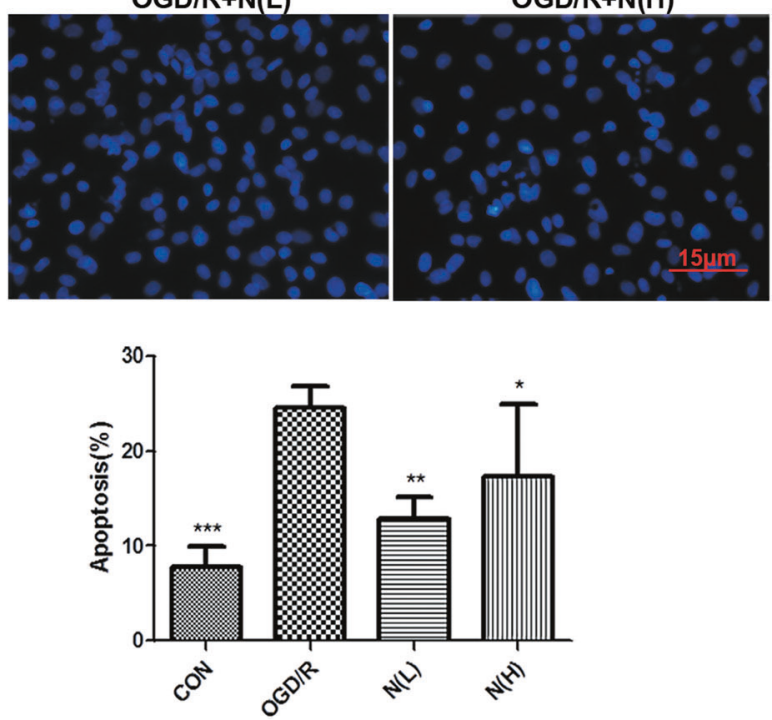

d
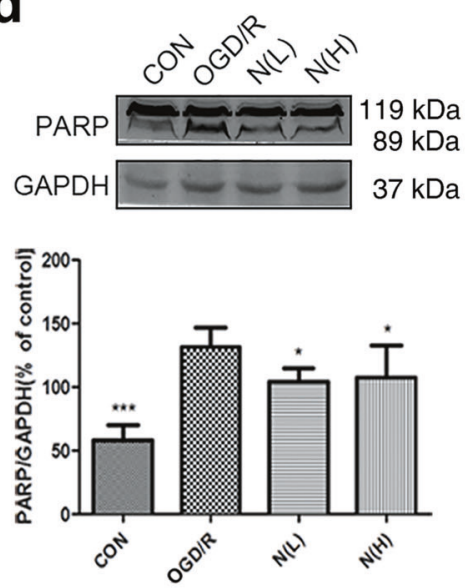

Fig. 4 NADPH inhibited cardiomyocyte apoptosis induced by OGD/R. Neonatal rat cardiomyocytes were treated as described in the legend of Fig. 2. $\mathrm{N}(\mathrm{L})=60 \mathrm{nM}, \mathrm{N}(\mathrm{H})=500 \mathrm{nM}$. a Representative graphs of cell apoptosis examined by flow cytometry. b Cardiomyocytes were stained with Hoechst 33342 and observed by fluorescence microscopy. Scale bar $=15 \mu \mathrm{m}$. c NADPH reduced the cleavage of caspase-3 in cardiomyocytes. d NADPH reduced the cleavage of PARP in cardiomyocytes. $n=3$ independent cultures. The bar represents the mean \pm SD, ${ }^{*} P<0.05,{ }^{* *} P<0.01,{ }^{* * *} P<0.001$ compared with the OGD/R group

supplementation with NADPH significantly reduced the myocardial infarct size induced by $\mathrm{I} / \mathrm{R}$ injury. In addition, NADPH administration reduced circulating LDH and cTnl levels. Similarly, in NRCMs subjected to OGD/R, NADPH treatment significantly increased cell viability and reduced cardiomyocyte injury. The in vivo and in vitro results both indicate that NADPH significantly inhibits myocardial I/R injury.

I/R injury leads to cardiomyocyte apoptosis, while prevention of cardiomyocyte apoptosis may be an effective strategy to protect against myocardial I/R injury [28, 29]. PARP, a DNA repair enzyme, is the cleavage substrate of caspase-3, the primary executor of apoptosis. The proteolytic cleavage of PARP through caspase- 3 is the prerequisite or early event in apoptosis [30]. Studies have shown that the cleavage of caspase- 3 and PARP mediates myocardial ischemic injuries [31]. Our results showed that NADPH administration in vitro and in vivo significantly reduced cardiomyocyte apoptosis induced by I/R injury, and NADPH reduced cleaved PARP and cleaved caspase- 3 . These results indicate that 
a

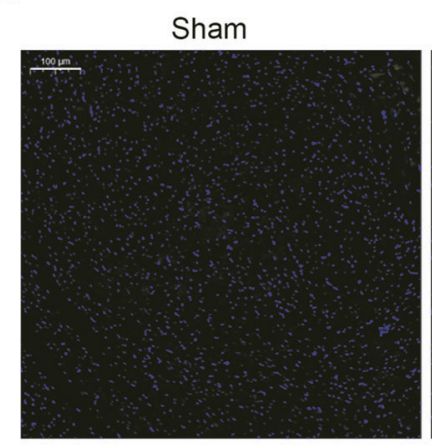

I/R

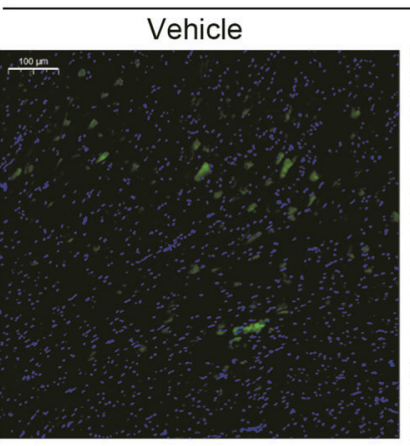

N16
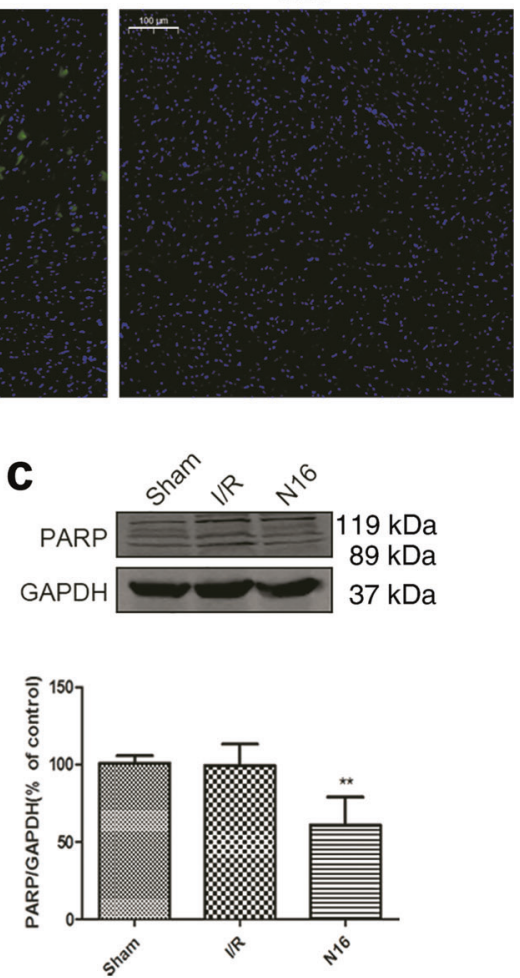

Fig. 5 NADPH inhibited cardiomyocyte apoptosis induced by myocardial I/R injury in rats. Rats were treated as described in the legend of Fig. 1. The left ventricle was dissected at $2 \mathrm{~h}$ after reperfusion and subjected to Western blotting. a Cardiomyocyte apoptosis was detected by TUNEL analysis. Scale bar $=100 \mu \mathrm{m}$. b NADPH reduced the cleavage of caspase-3 in the myocardium. $\mathbf{c}$ NADPH reduced the cleavage of PARP in the myocardium. $n=6$ rats. The bar represents the mean $\pm S D,{ }^{*} P<0.05,{ }^{* *} P<0.01$ compared with the I/R group

a

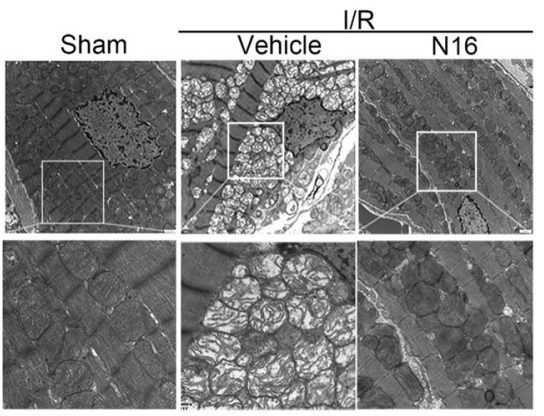

b
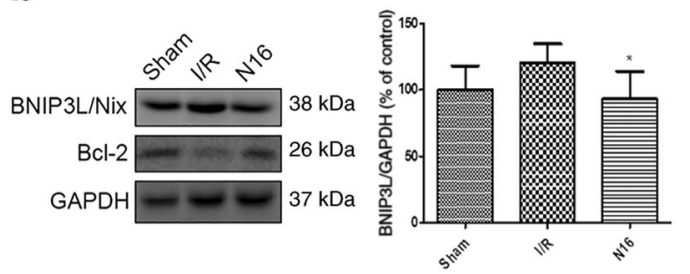

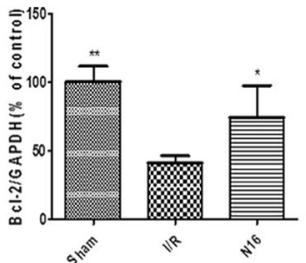

C
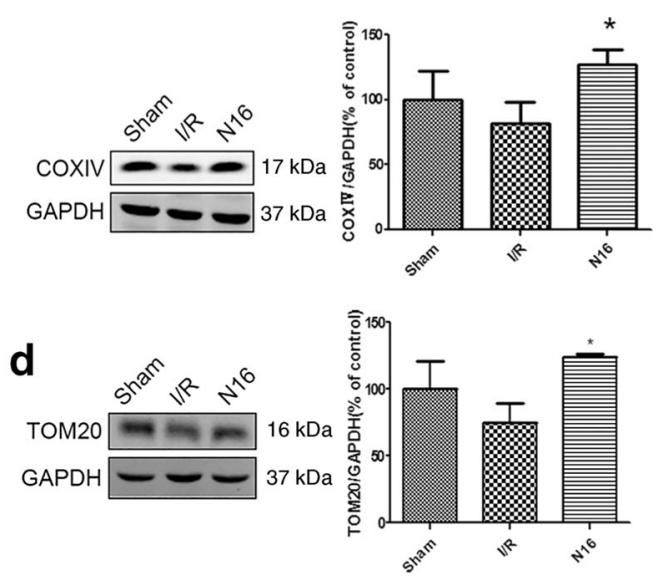

Fig. 6 NADPH ameliorated I/R-induced mitochondrial damage in rats. Rats were treated as described in the legend of Fig. 5. a Transmission electron microscopy analysis of mitochondrial morphology in the myocardium. Scale bar $=1 \mu \mathrm{m} . n=3$ rats. b NADPH reduced BNIP3L and increased $\mathrm{Bcl}-2$ in the myocardium. $\mathbf{c}$ NADPH increased COXIV in the myocardium. d NADPH increased TOM 20 in the myocardium. $n=6$ rats. The bar represents the mean \pm SD. ${ }^{*} P<0.05,{ }^{* *} P<0.01$ compared with the $1 / R$ group

NADPH can inhibit cardiomyocyte apoptosis induced by myocardial I/R injury.

I/R injury induced mitochondrial dysfunction in cardiomyocytes, resulting in decreased mitochondrial membrane potential and increased mitochondrial permeability. At the same time, mitochondria-localized $\mathrm{Bcl}-2$ expression is decreased, while proapoptotic proteins are increased to promote mitochondriadependent apoptosis [23]. In addition, BNIP3L is a mitochondrial protein that binds to $\mathrm{Bcl}-2$ and regulates mitochondrial function [32]. Studies have shown that mice lacking BNIP3 showed less 

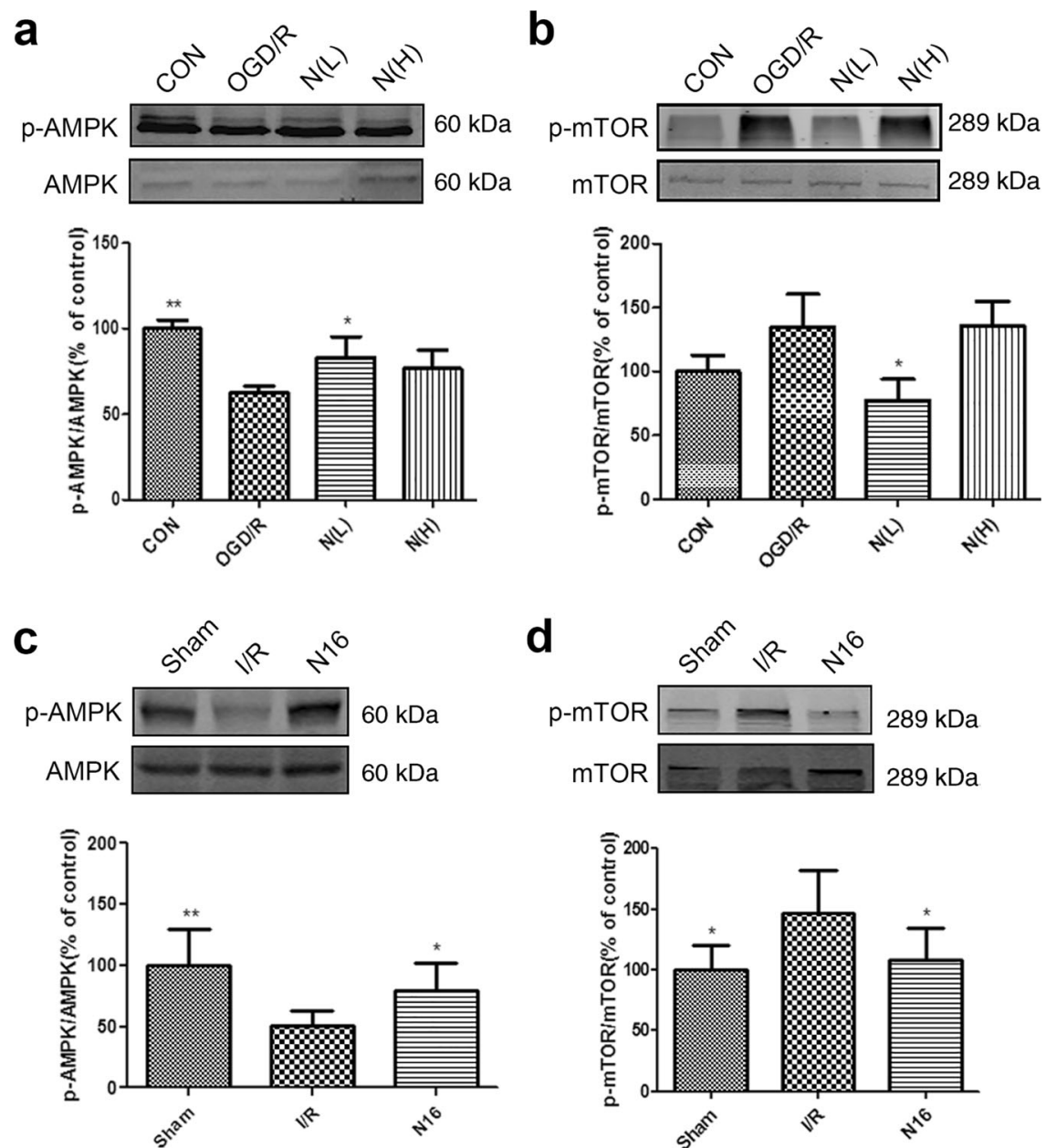

Fig. 7 NADPH activated the AMPK-mTOR pathway in myocardial ischemia/reperfusion injury. $\mathbf{a}$, b Neonatal rat cardiomyocytes were treated as described in Fig. 4. $\mathrm{N}(\mathrm{L})=60 \mathrm{nM}, \mathrm{N}(\mathrm{H})=500 \mathrm{nM}$. a NADPH increased the phosphorylation of AMPK in cardiomyocytes. b NADPH reduced the phosphorylation of mTOR in cardiomyocytes. $n=3$ independent cultures. $\mathbf{c}$, d Rats were treated as described in Fig. 5. c NADPH increased the phosphorylation of AMPK in the myocardium. $d$ NADPH reduced the phosphorylation of mTOR in the myocardium. $n=6$ rats. The bar represents mean $\pm S D,{ }^{*} P<0.05,{ }^{* *} P<0.01$ compared with the OGD/R group or I/R group

cardiomyocyte apoptosis and left ventricular remodeling after I/R [33]. In the present study, we found that in the myocardium undergoing $\mathrm{I} / \mathrm{R}$, mitochondria were severely damaged and vacuolated, mitochondrial membrane proteins COXIV and TOM20 were significantly reduced, BNIP3L expression was increased, and Bcl-2 expression was decreased, indicating that $\mathrm{I} / \mathrm{R}$ induces mitochondrial damage in the myocardium. However, NADPH administration can greatly reverse I/R-induced mitochondrial damage and apoptosis in cardiomyocytes, suggesting that NADPH may protect mitochondria to inhibit cardiomyocyte apoptosis induced by $\mathrm{I} / \mathrm{R}$.

The in vitro results showed that $20-500 \mathrm{nM}$ NADPH could protect primary cultured cardiomyocytes from OGD/R-induced injury. We then selected $60 \mathrm{nM}$ and $500 \mathrm{nM}$ NADPH for further mechanistic studies. Notably, $60 \mathrm{nM}$ NADPH inhibited cardiomyocyte apoptosis, while the effect of $500 \mathrm{nM}$ NADPH was relatively weak, suggesting that NADPH achieved the maximal effect at 60 nM. It is known that NADPH can be used as a substrate for NADPH oxidase (NOX) to produce reactive oxygen species (ROS) under certain stress conditions, and ROS are an important mediator involved in ischemia/reperfusion injury [11]. Therefore, we speculate that a high dose of NADPH may activate NOX to produce ROS, which partially offsets the cardioprotective effects of NADPH against myocardial ischemia/reperfusion injury.

AMPK is a serine/threonine protein kinase that responds to energy stress in cells [34]. AMPK is a heterotrimeric complex consisting of an a catalytic subunit and $\beta$ and $\gamma$ regulatory subunits. Phosphorylation of the a subunit site is required for its kinase activity. AMPK is activated by allosteric regulation of the subunits and an increased AMP/ATP ratio. Activated AMPK then shuts down the ATP depletion anabolic processes and initiates catabolism to correct the energy metabolism balance [34]. Studies have shown that activation of AMPK during myocardial ischemia-reperfusion is beneficial for cardiomyocyte survival, possibly due to the reduced apoptosis, increased ATP production and improved glucose and fatty acid metabolism [35, 36]. Dominant-negative mutations of AMPK impair glucose transport and fatty acid metabolism induced by ischemia, leading to increased susceptibility to cell damage and left ventricular dysfunction [37, 38]. In addition, AMPK has been shown to enhance mitochondrial function during I/R. In AMPK-inactivated hearts, mitochondrial ROS production is increased, while mitochondria show decreased resistance to mitochondrial permeability transition pores (mPTPs). Activation of AMPK prevents mitochondrial ROS production and inhibits MPTP opening during $l / R$, thereby protecting against mitochondrial damage and myocardial injury $[39,40]$.

In addition, mTOR is generally thought to be downstream of AMPK, which plays an important role in cell proliferation and growth [41]. Activation of AMPK downregulates mTOR activity, and AMPK participates in a variety of biological processes through 


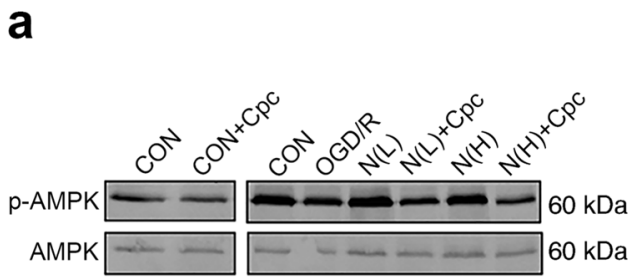

b

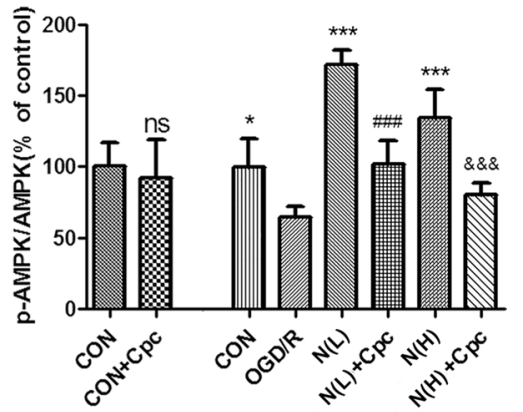

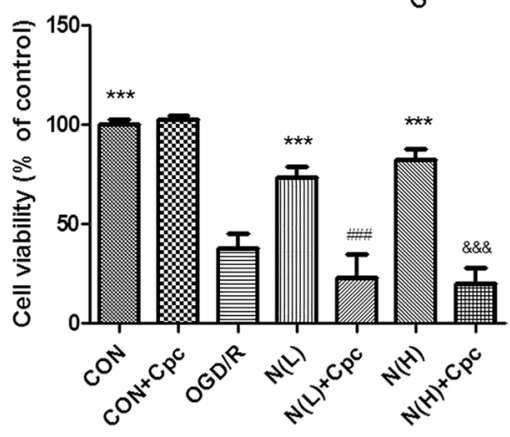

Fig. 8 Inhibition of AMPK by compound C partly reversed the protective effects of NADPH against myocardial I/R. Neonatal rat cardiomyocytes were treated as described in Figs. 1 and 4. $\mathrm{N}(\mathrm{L})=60 \mathrm{nM}, \mathrm{N}(\mathrm{H})=500 \mathrm{nM}$. Compound $\mathrm{C}(\mathrm{CpC}, 5 \mu \mathrm{M})$ was added to the medium at the onset of reperfusion. $\mathbf{a} \mathrm{CpC}$ reduced AMPK phosphorylation in cardiomyocytes. $\mathbf{b} \mathrm{CpC}$ abolished NADPH-induced protective effects in cardiomyocytes. The bar represents the mean $\pm \mathrm{SD}, n=3$ independent cultures. ${ }^{*} P<0.05,{ }^{* * * *} P<0.001$ compared with $\mathrm{OGD} / \mathrm{R}$, \#\#\# $P<0.001$ compared with $N(L)$, \&\&\& $P<0.05$ compared with $N(H)$

interacting with mTOR $[42,43]$. Previous studies have shown that oxidative stress activates the mTOR pathway, leading to apoptosis [44]. In addition, Chen et al. found that cadmium induced robust mTOR phosphorylation, while inhibition of mTOR phosphorylation by rapamycin prevented cadmium-induced apoptosis, suggesting that cadmium induces apoptosis by activating the mTOR pathway [45].

Consistent with the above studies, we found that myocardial I/R injury inhibited AMPK phosphorylation and increased mTOR phosphorylation both in vivo and in vitro, whereas exogenous NADPH activated AMPK phosphorylation and inhibited mTOR phosphorylation.

We next applied the widely used AMPK inhibitor compound $C$ [46] to determine whether NADPH protects against myocardial I/R injury through activating AMPK. The data showed that compound $C$ effectively reversed NADPH-induced AMPK phosphorylation. Compound $\mathrm{C}$ also abolished the protective effect of NADPH on myocardial I/R. These data suggest that NADPH may inhibit cardiomyocyte apoptosis induced by I/R injury by activating the AMPK/mTOR pathway. Several studies have found that AMPK agonists could protect against myocardial infarction. High doses of metformin protected the hearts of normal and diabetic mice from ischemic injury through the activation of cardiac AMPK [46]. A newly synthesized compound, A769662, which allosterically activates AMPK through interacting with the $\beta$ subunit, alleviated ischemia-reperfusion injury in in vitro perfused hearts and in the mouse coronary artery ligation reperfusion model [47]. AICAr, an AMPK activator, could reduce the myocardial infarct size in a rabbit model of ischemia-reperfusion injury [47]. These studies combined with our data implied that activators of the AMPKmTOR signaling pathway may mimic the protective effect of NADPH.

There are still some questions in this study that have not been clearly answered. First, although administration of exogenous NADPH significantly increased the NADPH concentration in both the serum and myocardium of rats, it is unclear whether the increased NADPH after administration is derived completely from exogenous administration because NADPH is an important endogenous biologically active substance. Isotopically labeled $\mathrm{NADPH}$ will be applied to answer the question in the future. Second, although the data showed that NADPH protects against myocardial $\mathrm{I} / \mathrm{R}$ injury through activation of the AMPK/mTOR pathway, the mechanism by which NADPH increases AMPK phosphorylation remains to be elucidated. AMPK activation involves the phosphorylation of Thr-172 in the a catalytic subunit, which has been widely demonstrated to be mediated by two upstream kinases, LKB1 and CaMKK $\beta$ (Ca/calmodulin-dependent protein kinase $\beta$ ) [48]. A recent study has shown that inhibition of G6PD activity and decreased NADPH levels can inhibit L-type Ca channel activity, while dialyzing NADPH into cells from a patch pipette solution increases intracellular L-type Ca channelmediated calcium influx [49]. Therefore, we assume that NADPH might increase intracellular calcium to activate CaMKK $\beta$, leading to the activation of AMPK. In addition, resveratrol has been reported to activate SIRT1 [48] or SIRT3 [50], which may then deacetylate and activate LKB1, resulting in AMPK phosphorylation and activation as well as mitochondrial function improvement. Therefore, we speculate that NADPH may also indirectly activate AMPK through the SIRT1(SIRT3)/LKB1 network. Finally, whether NADPH acts directly on a specific subunit of AMPK, leading to the activation of AMPK, is also an important subject worthy of further investigation. The exact mechanism by which NADPH activates AMPK will be the focus of our future research.

\section{CONCLUSION}

In summary, our results suggest that exogenous NADPH shows cardioprotective effects against myocardial I/R injury by activating the AMPK/mTOR pathway and inhibiting mitochondrial damage and apoptosis in cardiomyocytes. NADPH may become a potential candidate for the prevention and treatment of myocardial ischemic diseases. 


\section{ACKNOWLEDGEMENTS}

This work was supported by the National Natural Science Foundation of China (No.81673421, 81973315), Jiangsu Key Laboratory of Neuropsychiatric Diseases (BM2013003), Research Foundation from the Second Affiliated Hospital of Soochow University(SDFEYGJ1703), Young Medical Talents of Jiangsu Province (QNRC2016876), Health Talents Training Project of Gusu District(GSWS2019040), and Industrial Technology Innovation Project of Suzhou (SS201639, SS201763, SS201637). Medical Application Foundation of Suzhou (SYS2019074).

\section{AUTHOR CONTRIBUTIONS}

JZ and ZHQ designed research; YFW and XMC performed research; KQ performed research in the revision. PMC and JFC contributed new reagents or analytic tools; LWZ and PP analyzed data; YFW and RS wrote the paper. RS and HX revised the manuscript.

\section{ADDITIONAL INFORMATION}

Competing interests: The authors declare no competing interests.

\section{REFERENCES}

1. Writing Group M, Mozaffarian D, Benjamin EJ, Go AS, Arnett DK, Blaha MJ, et al. Heart disease and stroke statistics-2016 update: a report from the American heart association. Circulation. 2016;133:e38-360.

2. Chouchani ET, Pell VR, Gaude E, Aksentijevic D, Sundier SY, Robb EL, et al. Ischaemic accumulation of succinate controls reperfusion injury through mitochondrial ROS. Nature. 2014;515:431-5.

3. Zhang J, Karcz MK, Nadtochiy SM, Brookes PS. High-throughput screening reveals the mitochondrial complex I inhibitor nornicotine is cardioprotective in ischemia-reperfusion injury when delivered at reperfusion. Circ Res. 2015;117: A404.

4. Ying W. $\mathrm{NAD}^{+} / \mathrm{NADH}$ and $\mathrm{NADP}^{+} / \mathrm{NADPH}$ in cellular functions and cell death: regulation and biological consequences. Antioxid Redox Signal. 2008;10:179-206.

5. Burgoyne JR, Mongue-Din H, Eaton P, Shah AM. Redox signaling in cardiac physiology and pathology. Circ Res. 2012;111:1091-106.

6. Berger F, Ramirez-Hernandez $M H$, Ziegler $M$. The new life of a centenarian: signalling functions of NAD(P). Trends Biochem Sci. 2004;29:111-8.

7. Pollak N, Dolle C, Ziegler M. The power to reduce: pyridine nucleotides-small molecules with a multitude of functions. Biochem J. 2007;402:205-18.

8. Fessel JP, Oldham WM. Pyridine dinucleotides from molecules to man. Antioxid Redox Signal. 2018;28:180-212.

9. Seiler KS, Starnes JW. Exogenous GSH protection during hypoxia-reoxygenation of the isolated rat heart: impact of hypoxia duration. Free Radic Res. 2000;32:41-55.

10. Li M, Zhou ZP, Sun M, Cao L, Chen J, Qin YY, et al. Reduced nicotinamide adenine dinucleotide phosphate, a pentose phosphate pathway product, might be a novel drug candidate for ischemic stroke. Stroke. 2016;47:187-95.

11. Qin YY, Li M, Feng X, Wang J, Cao L, Shen XK, et al. Combined NADPH and the NOX inhibitor apocynin provides greater anti-inflammatory and neuroprotective effects in a mouse model of stroke. Free Radic Biol Med. 2017;104:333-45.

12. Huang Q, Sun M, Li M, Zhang D, Han F, Wu JC, et al. Combination of NAD(+) and NADPH offers greater neuroprotection in ischemic stroke models by relieving metabolic stress. Mol Neurobiol. 2018;55:6063-75.

13. Cao L, Zhang D, Chen J, Qin YY, Sheng R, Feng X, et al. G6PD plays a neuroprotective role in brain ischemia through promoting pentose phosphate pathway. Free Radic Biol Med. 2017;112:433-44.

14. Forini F, Nicolini G, Kusmic C, D'Aurizio R, Rizzo M, Baumgart M, et al. Integrative analysis of differentially expressed genes and miRNAs predicts complex T3mediated protective circuits in a rat model of cardiac ischemia reperfusion. Sci Rep. 2018;8:13870.

15. Aikawa R, Nawano M, Gu YP, Katagiri H, Asano T, Zhu WD, et al. Insulin prevents cardiomyocytes from oxidative stress-induced apoptosis through activation of PI3 kinase/Akt. Circulation. 2000;102:2873-9.

16. Akao M, Ohler A, O'Rourke B, Marban E. Mitochondrial ATP-sensitive potassium channels inhibit apoptosis induced by oxidative stress in cardiac cells. Circ Res. 2001;88:1267-75.

17. Nadtochiy SM, Redman E, Rahman I, Brookes PS. Lysine deacetylation in ischaemic preconditioning: the role of SIRT1. Cardiovasc Res. 2011;89:643-9.

18. Tseliou E, Kanazawa H, Dawkins J, Gallet R, Kreke M, Smith R, et al. Widespread myocardial delivery of heart-derived stem cells by nonocclusive triple-vessel intracoronary infusion in porcine ischemic cardiomyopathy: superior attenuation of adverse remodeling documented by magnetic resonance imaging and histology. PLoS One. 2016;11:e0144523.
19. Zhou JH, Zhang TT, Song DD, Xia YF, Qin ZH, Sheng R. TIGAR contributes to ischemic tolerance induced by cerebral preconditioning through scavenging of reactive oxygen species and inhibition of apoptosis. Sci Rep. 2016;6:27096

20. Reagan WJ, York M, Berridge B, Schultze E, Walker D, Pettit S. Comparison of cardiac troponin I and $\mathrm{T}$, including the evaluation of an ultrasensitive assay, as indicators of doxorubicin-induced cardiotoxicity. Toxicol Pathol. 2013;41:1146-58.

21. Apple FS, Murakami MM, Ler R, Walker D, York M; HESI Technical Committee of Biomarkers Working Group on Cardiac Troponins. Analytical characteristics of commercial cardiac troponin I and T immunoassays in serum from rats, dogs, and monkeys with induced acute myocardial injury. Clin Chem. 2008;54:1982-9.

22. Liu Y, Yang H, Liu LX, Yan W, Guo HJ, Li WJ, et al. NOD2 contributes to myocardial ischemia/reperfusion injury by regulating cardiomyocyte apoptosis and inflammation. Life Sci. 2016;149:10-7

23. Yasuda M, Han JW, Dionne CA, Boyd JM, Chinnadurai G. BNIP3alpha: a human homolog of mitochondrial proapoptotic protein BNIP3. Cancer Res. 1999;59:533-7.

24. Vega-Naredo I, Loureiro R, Mesquita KA, Barbosa IA, Tavares LC, Branco AF, et al. Mitochondrial metabolism directs stemness and differentiation in P19 embryonal carcinoma stem cells. Cell Death Differ. 2014:21:1560-74.

25. Yamamoto $\mathrm{H}$, Itoh N, Kawano S, Yatsukawa $\mathrm{Y}$, Momose $\mathrm{T}$, Makio T, et al. Dual role of the receptor Tom20 in specificity and efficiency of protein import into mitochondria. Proc Natl Acad Sci U S A. 2011;108:91-6.

26. Zhang $Y$, Wang B, Fu X, Guan S, Han W, Zhang J, et al. Exogenous $N A D(+)$ administration significantly protects against myocardial ischemia/reperfusion injury in rat model. Am J Transl Res. 2016;8:3342-50.

27. Reyes LA, Boslett J, Varadharaj S, De Pascali F, Hemann C, Druhan LJ, et al. Depletion of $\operatorname{NADP}(\mathrm{H})$ due to $\mathrm{CD} 38$ activation triggers endothelial dysfunction in the postischemic heart. Proc Natl Acad Sci U S A. 2015;112:11648-53.

28. Hsu CP, Zhai P, Yamamoto T, Maejima Y, Matsushima S, Hariharan N, et al. Silent information regulator 1 protects the heart from ischemia/reperfusion. Circulation. 2010;122:2170-82.

29. Pu J, Yuan A, Shan P, Gao E, Wang X, Wang Y, et al. Cardiomyocyte-expressed farnesoid-X-receptor is a novel apoptosis mediator and contributes to myocardial ischaemia/reperfusion injury. Eur Heart J. 2013;34:1834-45.

30. Virag L, Szabo C. The therapeutic potential of poly(ADP-ribose) polymerase inhibitors. Pharmacol Rev. 2002;54:375-429.

31. Piot CA, Martini JF, Bui SK, Wolfe CL. Ischemic preconditioning attenuates ischemia/reperfusion-induced activation of caspases and subsequent cleavage of poly(ADP-ribose) polymerase in rat hearts in vivo. Cardiovasc Res. 1999; 44:536-42.

32. Rikka S, Quinsay MN, Thomas RL, Kubli DA, Zhang X, Murphy AN, et al. Bnip3 impairs mitochondrial bioenergetics and stimulates mitochondrial turnover. Cell Death Differ. 2011;18:721-31.

33. Diwan A, Wansapura J, Syed FM, Matkovich SJ, Lorenz JN, Dorn GW 2nd, et al. Nix-mediated apoptosis links myocardial fibrosis, cardiac remodeling, and hypertrophy decompensation. Circulation. 2008;117:396-404.

34. Hardie DG. AMP-activated protein kinase: the guardian of cardiac energy status. J Clin Invest. 2004;114:465-8.

35. Qi D, Young LH. AMPK: energy sensor and survival mechanism in the ischemic heart. Trends Endocrinol Metabol. 2015:26:422-9.

36. Calvert JW, Gundewar S, Jha S, Greer JJ, Bestermann WH, Tian R, et al. Acute metformin therapy confers cardioprotection against myocardial infarction via AMPK-eNOS-mediated signaling. Diabetes. 2008;57:696-705

37. Xing Y, Musi N, Fujii N, Zou L, Luptak I, Hirshman MF, et al. Glucose metabolism and energy homeostasis in mouse hearts overexpressing dominant negative alpha2 subunit of AMP-activated protein kinase. J Biol Chem. 2003;278:28372-7.

38. Russell RR 3rd, Li J, Coven DL, Pypaert M, Zechner C, Palmeri M, et al. AMPactivated protein kinase mediates ischemic glucose uptake and prevents postischemic cardiac dysfunction, apoptosis, and injury. J Clin Invest. 2004; 114:495-503.

39. Paiva MA, Rutter-Locher Z, Goncalves LM, Providencia LA, Davidson SM, Yellon DM, et al. Enhancing AMPK activation during ischemia protects the diabetic heart against reperfusion injury. Am J Physiol Heart Circ Physiol. 2011;300:H2123-34.

40. Zaha VG, Qi D, Su KN, Palmeri M, Lee HY, Hu X, et al. AMPK is critical for mitochondrial function during reperfusion after myocardial ischemia. J Mol Cell Cardiol. 2016;91:104-13.

41. Bjornsti MA, Houghton PJ. The TOR pathway: a target for cancer therapy. Nat Rev Cancer. 2004;4:335-48.

42. Sciarretta $S$, Volpe $M$, Sadoshima J. Mammalian target of rapamycin signaling in cardiac physiology and disease. Circ Res. 2014;114:549-64.

43. Ding WX, Ni HM, Gao W, Hou YF, Melan MA, Chen X, et al. Differential effects of endoplasmic reticulum stress-induced autophagy on cell survival. J Biol Chem. 2007;282:4702-10. 
44. Chen L, Xu B, Liu L, Luo Y, Zhou H, Chen W, et al. Cadmium induction of reactive oxygen species activates the mTOR pathway, leading to neuronal cell death. Free Radic Biol Med. 2011;50:624-32.

45. Chen $S$, Ren $Q$, Zhang J, Ye $Y, Z$ hang $Z, X u Y$, et al. $N$-acetyl-L-cysteine protects against cadmium-induced neuronal apoptosis by inhibiting ROS-dependent activation of Akt/mTOR pathway in mouse brain. Neuropathol Appl Neurobiol. 2014;40:759-77

46. Zhou G, Myers R, Li Y, Chen Y, Shen X, Fenyk-Melody J, et al. Role of AMPactivated protein kinase in mechanism of metformin action. J Clin Invest. 2001;108:1167-74.

47. Hardie DG. AMP-activated protein kinase: an energy sensor that regulates all aspects of cell function. Genes Dev. 2011;25:1895-908.
48. Price NL, Gomes AP, Ling AJ, Duarte FV, Martin-Montalvo A, North BJ, et al. SIRT1 is required for AMPK activation and the beneficial effects of resveratrol on mitochondrial function. Cell Metab. 2012;15:675-90.

49. Rawat DK, Hecker P, Watanabe M, Chettimada S, Levy RJ, Okada T, et al. Glucose6-phosphate dehydrogenase and NADPH redox regulates cardiac myocyte L-type calcium channel activity and myocardial contractile function. PLoS One. 2012;7: e45365.

50. Duan WJ, Li YF, Liu FL, Deng J, Wu YP, Yuan WL, et al. A SIRT3/AMPK/autophagy network orchestrates the protective effects of trans-resveratrol in stressed peritoneal macrophages and RAW264.7 macrophages. Free Radic Biol Med. 2016:95:230-42. 